\title{
An alternative to the variation of the fine structure constant
}

\author{
M. E. DE SOUZA \\ Departamento de Física, Universidade Federal de Sergipe, Campus Univer- \\ sitário, 49100-000 São Cristóvão, Sergipe, Brazil - e-mail: mdesouza@ufs.br \\ Considering that quasars evolve into normal galaxies the average ex- \\ pansion velocity of matter in quasars (or galaxies) can be estimated \\ approximately. Such velocity makes us to see the light of quasars \\ slightly Doppler shifted which explains the misleading variation of \\ the fine structure constant.
}

There have been reports ${ }^{1-4}$ in the literature proposing a time variation of the fine structure constant. They are based on observations of absorption lines of gas clouds seen against background quasars. Thus the clouds are quasar absorbers. This supposed time variation of $\alpha$ motivated the recent and very important article of Davies ${ }^{5}$ which proposes that the velocity of light may vary with time. This would cause profound changes in the foundatins of physics.

This work presents an alternative explanation for the phenomenon observed in the gas clouds which maintains $\alpha$ as a fundamental constant of nature, that is, independent of time. It is based on a proposal for the evolution of galaxies from quasars. The remarkable work of Márquez et al. ${ }^{6}$ has shown that very high redshift elliptical galaxies appear to harbor quasars. They have also shown that such galaxies are very small and have diameters smaller than $1 \mathrm{kpc}$. All the studied objects (about 15 quasars) have extended structures of ionized gas around them (this fact had already been presented by other researchers). They have found other galaxies in the fields of the studied objects only a few kpc away from them. Some of the quasars present asymmetric radio sources with collimated one-sided jets of extended ionized gas. This means that galaxies are born as quasars that become galaxies by means of the shedding of matter (ionized gas) from their cores. The same kind of phenomenon has been observed in galaxies. Very recent data ${ }^{7}$ of NGC 6240 , which is considered a typical protogalaxy, show that "approximately $70 \%$ of the total radio power at $20 \mathrm{~cm}$ originates from the nuclear region $(\leq 1.5 \mathrm{kpc})$, of which half is emitted by two unresolved $(\mathrm{R} \leq 30 \mathrm{pc})$ cores and half by a diffuse component. Nearly all of the other $30 \%$ of the total radio power comes from an arm-like region extending westward from the nuclear region". A very important property of many quasars is their brightness which can vary from night to night. This flickering may have its origin in the outward motion of large quantities of matter from their cores. This brightness variability is also present in Seyfert galaxies which are powerful sources of infrared radiation. Many of them are also strong radio emitters. For example, over a period of a few months, the nucleus of the Seyfert galaxy M77(or 
NGC1068) switches on and off a power output equivalent to the total luminosity of our galaxy ${ }^{8}$. It is also worth noting that the nuclei of Seyfert galaxies are very bright and have a general starlike appearance. Researchers have found that some Seyfert galaxies exhibit explosive phenomena ${ }^{8}$. For example, M77 and NGC4151 expel huge amounts of gas from their nuclei. The spectra of both galaxies show strong emission lines, just as quasars'. Shaver et al. ${ }^{9}$ have found that there is almost no quasar for $z<0.5$. This clearly shows that quasars evolve into galaxies.

This ejection of matter is quite common in active galaxies also. Let us mention just some of them. NGC 2992 presents a jet-like structure and a circum-nuclear ring $^{10}$. Falcke and Biermann ${ }^{11}$ report that there is a large scale emission-like jet going outward from the core of NGC 4258 with a mass of about $4 \times 10^{35} \mathrm{~kg}$ and with a kinetic power of approximately $10^{42} \mathrm{ergs} / \mathrm{s}$ and expansion velocity of $2000 \mathrm{~km} / \mathrm{s}$. This is of the same order of supernovae velocities. It is well known that BL Lacertae objects are powerful sources of radio waves and infrared radiation. They share with quasars the fact of exhibiting a starlike appearance and of showing short-term brightness fluctuations. As some quasars do, they also have a nebulosity around the bright nucleus. Researchers ${ }^{12}$ have managed to obtain the spectrum of their nebulosity. The spectrum of the nebulosity is strikingly similar to the spectrum of an elliptical galaxy(M32's spectrum, in this case). In terms of the evolution above described they are simply an evolutionary stage of a quasar towards becoming a more quiet galaxy. Radio galaxies share with BL Lacertae objects many of the properties of quasars. As Heckman et al. ${ }^{12}$ have shown, in the middle and far infrared (MFIR) quasars are more powerful sources of MFIR radiation than radio galaxies. Also, there have been investigations showing that the emission from the narrow-line region(NLR) in radioloud quasars is stronger than in radio galaxies of the same radio power ${ }^{13,14,15}$. Goodrich and Cohen ${ }^{16}$ have studied the polarization in the broad-line radio galaxy $3 \mathrm{C}$ 109. After the intervening dust is taken into account the absolute V-magnitude of this galaxy becomes -26.6 or brighter, which puts it in the quasar luminosity range. The investigators suggest that "many radio galaxies may be quasars with their jets pointed away from our direct line of sight". It has also been established that radio galaxies are found at intermediate or high redshifts and that they are clearly related to galactic evolution because as the redshift increases cluster galaxies become bluer on average, and contain more young stars in their nuclei. This is also valid for radio galaxies: the higher the redshift, the higher their activity. All these data show that a radio galaxy is just an evolutionary stage of an active galaxy towards becoming a quiet galaxy, i.e., it is just a stage of the slow transformation by means of an overall expansion of a quasar into a normal galaxy.

In the light of the above considerations the nuclei of old spirals must exhibit a moderate activity. This is actually the case. The activity must be inversely proportional to the galaxy's age, i.e., it must be a function of luminosity. The bluer they are, the more active their nuclei must be. As discussed above there must also exist a relation between this activity and the size of the nucleus(as compared to the disk) in spiral galaxies. Our galaxy has a mild activity at its 
center. Most of the activity is concentrated in a region called Sagittarius A, which includes the galactic center. It emits synchroton and infrared radiations. Despite its large energy output Sagittarius A is quite small, being only about 40 light years in diameter. Besides Sagittarius A our galaxy exposes other evidences showing that in the past it was a much more compact object: a) Close to the center, on opposite sides of it, there are two enormous expanding arms of hydrogen going away from the center at speeds of $53 \mathrm{~km} / \mathrm{s}$ and $153 \mathrm{~km} / \mathrm{s}$; b) Even closer to the center there is the ring called Sagittarius B2 which is expanding at a speed of $110 \mathrm{~km} / \mathrm{s}^{(8,17)}$. It is worth noting that the speeds are low(as compared to the velocities of relativistic electrons from possible black holes). This phenomenon is not restricted to our galaxy. Recent high-resolution molecular-line observations of external galaxies have revealed that galactic nuclei are often associated with similar expanding rings ${ }^{18}$.

This paper will not deal with the mechanism of the expulsion of matter from the center of quasars and will be restricted to showing what may be happening in the case of the clouds absorption lines. It is well known that the size of a quasar is smaller than $1 \mathrm{kpc}$ and that a typical galaxy has a radius of about 50.000 light-years $\approx 5 \times 10^{20} \mathrm{~m}$ and, as the age of the Universe is about $10^{17} \mathrm{~s}$, the average expansion velocity of matter in galaxies since the time when they were quasars is of the order of

$$
<V_{e}>\approx 5 \times 10^{3} \mathrm{~m} / \mathrm{s} .
$$

This figure is just a rough estimate. For very high redshift quasars the expansion speed was at least an order of magnitude higher as it is inferred from the data on shedding of matter from the cores of quasars and galaxies. Therefore, the light of quasars should be Doppler shifted to higher frequencies for the light that is coming from points close to the line of sight. This means that the quasar light that reaches a gas cloud is slightly Doppler shifted due to the expansion of matter with respect to a rest-frame sitting in the center of the quasar. The shift is given by

$$
\frac{\Delta \nu}{\nu_{0}}=-\frac{<V_{e}>}{c} \approx-1.67 \times 10^{-5}
$$

which is of the same order of magnitude of the shifts attributed to variation of the fine structure constant around $z=1$ in the work of Webb et al. ${ }^{4}$. Take notice that if we attribute the above result to a variation of the fine structure function we obtain

$$
\frac{\Delta \alpha}{\alpha}=\frac{1}{2} \frac{\Delta \nu}{\nu_{0}}=-0.835 \times 10^{-5}
$$

which is a figure of the order of magnitude of that found in Webb et al. ${ }^{4}$. Thus, comparing results from quasars at two different redshifts $z_{1}$ and $z_{2}$ (with $z_{1}>$ $\left.z_{2}\right)$, since more active quasars have higher redshifts, it is expected $\left\langle V_{e}\right\rangle(z=$ $z_{1}$ ) to be larger than $\left\langle V_{e}\right\rangle\left(z=z_{2}\right)$ and, of course, $\left|\Delta \nu\left(z_{1}\right)\right|>\left|\Delta \nu\left(z_{2}\right)\right|$. For very high redshift quasars $\frac{\Delta \nu}{\nu_{0}}$ may even reach $10^{-4}$ or $10^{-3}$ which is in line with the upper limit of Cowie et al. ${ }^{19}$ according to whom $\left|\frac{\Delta \alpha}{\alpha}\right|<3.5 \times 10^{-4}$ for $z \sim 3$. 
Therefore, the observations of the variation of the fine structure constant may reveal, actually, how quasars evolve towards becoming quiet, normal galaxies. And thus, the foundations of physics continue being solid as ever!

References

1. Marciano, W. Phys. Rev. Lett. 52, 489 (1984)

2. Barrow, J. D. Phys. Rev. D 35, 1805 (1987)

3. Damour, T. and Polyakov, A. M. Nucl. Phys. B423, 532 (1994)

4. Webb, J. K. et al. Phys. Rev. Lett. 82, 884 (1999)

5. Davies, P. C. W. et al. Nature 418, 603 (2002)

6. Márquez, I. et al. astro-ph/9810012.

7. Colbert, E. J. M. et al. in The Radio Emission from the Ultra-Luminous Far-Infrared Galaxy NGC 6240, astro-ph/9405046

8. Kaufmann,III, W. J. in Galaxies and Quasars (W.H.Freeman and Company, San Francisco, 1979).

9. Shaver, P. A. et al. astro-ph9801211.

10. Chapman, S. C. et al. astro-ph/9810250.

11. H. Falcke, H., Biermann, P. L. astro-ph/9810226

12. Heckman, T. M. et al. Ap.J., 391, 39 (1992).

13. Baum, S, Heckman, T. M. Astrophys. J 336, 702 (1989).

14. Jackson, N, Browne I. Nature, 343, 43 (1990).

15. Lawrence, A. Mon. Not. R. Astr. Soc., 1992.

16. Goodrich, R. W., Cohen, M. H. Astrophys. J. 391, 623 (1992).

17. Sofue, Y. Astro. Lett. Comm. 28, 1 (1990).

18. Nakai, N. et al. Pub. Astr. Soc. Japan 39, 685 (1987).

19. Cowie, L. L., Songalia, A. Astrophys. J. 453, 596 (1995) 